\title{
Generation of Radio Noise in the Vicinity of the Earth
}

\author{
P. A. Sturrock
}

\author{
Contribution From W.W. Hansen Laboratories of Physics, Stanford University, Stanford, California
}

(Received August 18, 1961)

\begin{abstract}
A tentative classification of possible sources of radio noise in the vicinity of the earth may be obtained by examining separately available sources of power and known mechanisms for conversion of this power. Among the former we may list high-energy electrons such as those trapped in the Van Allen belts; the solar wind; bursts of high-energy particles ejected by the sun; shock waves in the interplanetary medium originating on the sun; and the rotational energy of the earth. Mechanisms of conversion may be classified as "direct," such as synchrotron and Cerenkov radiation, and "indirect." Indirect conversion involves the excitation of an intermediate state by the available sources of power and subsequent radiation by this state. This intermediate state may be localized heating, the formation of unstable current patterns, the acceleration of particles, or the generation of waves which are themselves non-radiative, such as plasma oscillations. The following mechanisms which are relevant to the generation of radio noise receive special attention: Cerenkov radiation, two-stream instability, and the coupling of waves by inhomogeneity and nonlinearity.
\end{abstract}

\section{Introduction}

Some of the non-manmade radio noise which is received on the earth is due to lightning discharges and similar phenomena in the earth's atmosphere. Radio noise also reaches the earth from many astronomical bodies. In this article, the question will be raised as to whether any radio noise received on earth is generated in the vicinity of the earth by mechanisms taking place outside of the earth's atmosphere.

The generation of radio noise may be regarded as the transfer of energy from a "source" into electromagnetic waves. One may therefore classify possible schemes for the generation of radio noise by listing possible sources of energy and possible mechanisms for the conversion of this energy into electromagnetic waves.

Among the sources of energy which come to mind are the following:

1. High-energy electrons in the Van Allen belts

[Van Allen, 1959].

2. The solar wind [Parker, 1958].

3. Bursts of high-energy particles ejected by the sun [Meyer, Parker, and Simpson, 1958].

4. Shock waves in the interplanetary medium originating on the sun [Gold, 1959].

5. Rotational energy of the earth.

Conversion mechanisms may be divided into two types which we term "direct" and "indirect". Direct mechanisms convert energy from the form it has in the source to that of electromagnetic waves without going through any intermediate state. Cerenkov radiation and synchrotron radiation are examples of direct mechanisms, if the source of energy is taken to be the kinetic energy of the charged particles. The intermediate processes involved in indirect conversion mechanisms may be, for instance, the heating

\footnotetext{
1 The work reported in this paper was sponsored by the Air Force Office of Scientific Research of the Air Research and Development Command, under Contract A F 49(638)-342.

${ }_{2}$ An earlier version of this paper was presented at the 1961 Spring Meeting of ${ }^{2}$ An earlier version of this paper was presented at the 1961 Spring Meeting of
the International Scientific Radio Union (URSI), Washington, D.C. (May 1961).
}

of an ionized gas, the acceleration of charged particles, or the excitation of waves or oscillations other than electromagnetic waves.

Before considering the various combinations of source plus conversion mechanisms which may be relevant to the problem in hand, we shall discuss briefly certain ideas of plasma physics which are helpful in analyzing the present problem. In section 2, we shall discuss Cerenkov radiation in order to show that, for the case of a "point source" or "beam source," the group velocity of the waves has an important bearing on the efficiency of the conversion mechanism. In section 3 , it will be shown that the familiar two-stream electrostatic instability [Bohm and Gross, 1949] is one member of a general class of two-stream instabilities, other members of which may be important for the problem in hand. In section 4 , we consider ways in which energy may be fed from one type of wave to another, for instance, from plasma oscillations into electromagnetic waves. In section 5, we consider some of the ways in which radio noise might arise from the sources listed above.

\section{Resonant Cerenkov Radiation}

Consider, for simplicity, a beam of particles uniform in the $y$ and $z$ directions, confined between the planes $x=0$ and $x=a$, and traveling with velocity $v$ in the $z$ direction. Inhomogeneities of the beam give rise to the excitation of waves in the medium through which the beam passes. Hence the beam may be represented by an appropriate source term in the wave equation. In consequence, the energy equation, as applied to the energy of excited waves, takes the form

$$
\frac{\partial E}{\partial t}+\Delta \cdot \mathbf{S}=F A \cos \phi
$$

where $E$ is energy density, $\mathbf{S}$ is energy flow, $A$ is the 
amplitude of the excited wave, $F$ denotes the strength of the source, and $\phi$ is the phase angle between the amplitude "vector" and the force "vector". For present purposes, we neglect dissipation of energy by collisions and radiation.

Now consider a steady-state situation and apply the above equation to waves of one particular wavevector $\mathbf{k}$ and frequency $\omega$ for the favorable case that the force and amplitude are in phase everywhere. The last condition leads, in particular, to the selection rule

$$
\omega-\mathbf{v} \cdot \mathbf{k}=0 \text {. }
$$

With these assumptions, and the additional simplification that the measure of amplitude is one for which

$$
E=A^{2},
$$

(2.1) reduces to

$$
u_{x} \frac{d A}{d x}=\frac{1}{2} F,
$$

where we have used the familiar relation [Brillouin, 1960]

$$
\mathbf{S}=E \mathbf{u}
$$

between energy density and energy flow, u being the group velocity of the wave.

If the group velocity component $u_{x}$ is positive, we expect that $A=0$ for $x<0$, so that

$$
A(x)=\frac{1}{2 u_{x}} \int_{0}^{x} F\left(x^{\prime}\right) d x^{\prime} .
$$

We now see that the wave excitation mechanism which we are considering is resonant when $u_{x}=0$, which is understandable since there is then no loss of energy from the region of wave excitation by the convection mechanism associated with group velocity.

In case of a beam which is of finite extent in both transverse directions, we should term the process "resonant" if the group velocity has no transverse component. In the case of a moving source finite in all dimensions, we see that the condition for resonance would be

$$
\mathbf{u}=\mathbf{v},
$$

that is, that the group velocity is identical with the source velocity. Note, from (2.2), that it is not necessary that the phase velocity be identical with the source velocity.

It appears that the above resonance condition is important in the mechanism of Type II solar radio bursts [Sturrock, 1961a], so that one might expect it to be important also in any similar processes which take place in the vicinity of the earth.

\section{Generalized Two-Stream Instability}

The concept of stability plays an important role in any study of energy conversion. Instability may be regarded as the cumulative transfer of energy from one form into another, so that, in looking for mechanisms for the generation of electromagnetic waves, one should pay special attention to possible instabilities of the system under consideration. The familiar two-stream electrostatic instability $[\mathrm{Bohm}$ and Gross, 1949] is a particularly interesting example, since it is a mechanism for the conversion of the kinetic energy of a stream of charged particles into oscillation energy. The purpose of this section is to point out a generalization of this two-stream mechanism, whereby the kinetic energy of particle streams can be converted into energy of excitation of waves of various types.

Consider two wave-propagating systems, one of which we regard as stationary and the other of which we regard as moving with velocity $v$ in the $z$-direction. Suppose, for present purposes, that coupling between the systems is weak. Then there will be significant interaction only if both systems can support waves of the same frequency $\omega$ and wave number $k$, assuming now that the wave vectors are directed along the $z$-axis. In the moving medium, the frequency and wave number of such a wave will be $\omega^{\prime}$, $k^{\prime}$, where

$$
\omega^{\prime}=\omega-\imath k, k^{\prime}=k .
$$

Suppose that the combined system is unstable to disturbances of the wave number being considered. Then the amplitude of the two waves will grow (slowly, because the coupling is assumed to be small). For small amplitudes, energy associated with the disturbance will be proportional to the square of the amplitude, which is now assumed to be growing exponentially. However, energy associated with the disturbance is a conserved quantity. A function which is constant and which varies exponentially must be identically zero. Hence the energy to be associated with the two interacting waves is zero, which is possible only if the energy of one wave is positive and that of the other wave is negative.

The sense in which one may ascribe negative energy to a wave has been discussed elsewhere [Sturrock, 1960]. Assuming that, in a coordinate system at rest with respect to the medium, the wave energy is positive, the wave energy will appear to be negative to an observer moving at such a velocity that the direction of the phase velocity of the wave is reversed. Hence, in the above case, the wave in the moving medium would appear to have negative energy provided that

$$
-v<\omega^{\prime} / k^{\prime}<0 \text { or } 0<\omega^{\prime} / k^{\prime}<-v .
$$

If, for any coordinate system (and if it is true for one, it is true for all coordinate systems), we find that waves in two different media have the same frequency, the same wave number, but energies of opposite signs, we may conclude that this system is unstable. This situation arises when the relative velocity of the media is in magnitude equal to the sum of the magnitudes of the phase velocities in the two media. This mechanism may be referred to as "generalized two-stream instability." 
Consideration of the above mechanism will enable one to determine what waves of a plasma may be excited to high amplitudes by means of a stream of particles of appreciable density. It also enables one to determine what waves may be excited at an interface between two media which are moving with different velocities. One may regard Helmholtz instability [Lamb, 1930] as a special case of generalized two-stream instability.

\section{Wave-Coupling Mechanisms}

We first note that, in the linear approximation, there is no coupling between waves in a uniform medium since these waves are, by definition, dynamical normal modes of the system. This statement immediately suggests two ways of obtaining coupling between waves: if the small amplitude restriction or the requirement of homogeneity is relaxed, we might expect to obtain coupling which would then be attributed to nonlinearity or inhomogeneity.

Let us first consider the possibility of interaction between three waves, the frequencies and wave vectors of which are $\omega_{1}, \mathbf{k}_{1}, \omega_{2}, \mathbf{k}_{2}$ and $\omega_{3}, \mathbf{k}_{3}$. We expect the interaction to be significant only if an appropriate phase relation is maintained between the waves over large distances and large times. This means that the frequencies and wave numbers must satisfy selection rules such as the following:

$$
\omega_{1}+\omega_{2}=\omega_{3}, \mathbf{k}_{1}+\mathbf{k}_{2}=\mathbf{k}_{3} .
$$

The larger the number of waves involved in such a selection rule, the higher is the power of the amplitudes on which the process depends, so that-for weak excitation of the medium - the weaker is the resulting coupling.

In interpreting the above formulas, note that more than one wave may be of the same type. In (4.1), for instance, waves 1 and 2 could be plasma oscillations in a simple isotropic plasma, while wave 3 could be an electromagnetic wave in this plasma. We then see that plasma oscillations in a uniform plasma can radiate, the dominant frequency being $2 \omega_{p}$, i.e., twice the plasma frequency [Sturrock, 1961b].

It is of course true that selection rules such as (4.1) do not guarantee coupling but are merely prerequisites for coupling. It is in addition necessary that the "interaction energy" be nonzero, that is, that the energy density (from which one may form the Hamiltonian) should contain a term involving the amplitudes of the waves in a form related to the selection rule.

Wave-coupling by inhomogeneities may be regarded as a special case of nonlinear coupling between waves, since, if the inhomogeneity is slight, it may be Fourier analyzed into a number of "waves" of zero frequency. With this minor change, selection rules such as (4.1) still apply.

To the above two mechanisms, we may add a third, which is even more familiar, but which should perhaps be referred to as a "conversion mechanism" rather than a "coupling mechanism": this is the "adiabatic mechanism." If a wave of a certain type is being propagated in a stationary medium, the parameters of which are changing slowly with distance, the wave will continue, with the same frequency and the same power (ignoring absorption), but the phase velocity will adjust itself to the changing parameters. We neglect, for simplicity, the possibility that the parameters may at some point cause the wave to be nonpropagating.

The reason that this mechanism is of particular interest here is that it permits Cerenkov radiation from a beam of particles, for which the phase velocity is necessarily less than the speed of light, to be converted very efficiently into normal electromagnetic radiation traveling with the speed of light. If, for instance, the extraordinary wave [Spitzer, 1956] is excited in a plasma permeated by a magnetic field, and if the density of the plasma decreases slowly to zero, this wave will emerge as a normal electromagnetic wave, provided that no resonant frequencies, such as the plasma frequency or gyro-frequency, cross the wave frequency.

\section{Possible Mechanisms of Noise Generation}

We now review the sources of energy listed in section 1, and look for mechanisms by which part of this energy could be converted into radio noise.

Consider first of all the energy source represented by the high-energy electrons stored in the Van Allen belts. Clearly, these electrons can generate radio noise by the direct mechanism of cyclotron (or "synchrotron") radiation. We adopt the estimate of Van Allen [1959] that the outer belt contains 10 electrons per $\mathrm{cm}^{3}$ with energy in excess of $20 \mathrm{kv}$, and make a conservative estimate by giving all electrons energy equal to this lower limit. The volume of the belt is approximately $3.10^{28} \mathrm{~cm}^{3}$ and, since the height is approximately $2.5 R_{E}$, the magnetic field strength is approximately 0.02 gauss. A conservative estimate of the intensity is obtained by ascribing the total radiated power to an area of $4 \pi\left(2.5 R_{E}\right)^{2}$ and a solid angle of $4 \pi$. If we assume that, because of variation of magnetic field strength over the volume of the belt, the radiation covers a band of $30 \mathrm{kc} / \mathrm{s}$ centered at $60 \mathrm{kc} / \mathrm{s}$, we find that the radiation intensity is approximately $2.10^{-18} \mathrm{watt} / \mathrm{m}^{2}$ steradian $\mathrm{c} / \mathrm{s}$, corresponding to a brightness temperature of 40,000 degrees. Although this is a substantial intensity, this radiation would be unobservable over most of the earth's surface because it is shielded by the ionosphere. It is possible that some fraction of this noise would arrive at the auroral zones.

Consider next the solar wind [Parker, 1958]. One would not expect radiation by a direct mechanism, but radio noise might be genera ted by an indirect mechanism, since instabilities of the generalized twostream variety may be expected to arise when the solar wind impinges upon the earth's magnetosphere. Among the various wave types which might participate in this instability are plasma oscillations, magnetohydrodynamic waves and acoustic waves, 
possibly in the form of ion-acoustic waves. None of these is a radiative (i.e., an electromagnetic) wave, so that generation of radio noise requires the operation of a wave-coupling mechanism. Inhomogeneity or nonlinearity might provide such coupling. It should be noted that if the extraordinary mode, perhaps in the form known as the whistler mode [Storey, 1953] participates in the instability, radio noise might be propagated to the earth's atmosphere as a "whistler." Since whistlers appear to couple quite efficiently to electromagnetic waves in the earth's atmosphere, this would be expected to give higher intensities. On the other hand, the transmission of whistler waves from the surface of the magnetosphere to the surface of the earth might be impossible, or possible only for certain regions of the earth's surface.

The third possible source of energy to be considered comprises bursts of high-energy particles ejected by the sun. Since such particles may enter the earth's magnetosphere, there is the possibility of direct generation of radio noise by the mechanism of Cerenkov radiation. If the extraordinary mode, of frequency above the plasma frequency, is excited in this way, the noise would appear at the earth's surface as normal electromagnetic radiation by the process of adiabatic conversion.

The possibility of similar excitation of whistler modes has been considered by Gallet and Helliwell [1959]. Dr. N. R. Brice (personal communication) has shown in this connection that the condition for resonant Cerenkov radiation can indeed be satisfied. From the dispersion relation

$$
\omega=\frac{c^{2} k^{2} \omega g \cos \theta}{\omega_{p}^{2}+c^{2} k^{2}}
$$

where $\omega_{g}$ and $\omega_{p}$ are the gyro- and plasma-frequencies, $k$ is the magnitude of the wave vector, and $\theta$ is its inclination to the magnetic field, one finds that the condition that the group velocity should be parallel to the direction of the magnetic field, which we assume to be also the direction of travel of charged particles, is that

$$
k=\omega_{p} / c .
$$

One then finds that the frequency of a wave depends upon the direction of its phase velocity as follows,

$$
\omega=\frac{1}{2} \omega_{g} \cos \theta
$$

and that the magnitude of the group velocity is now independent of $\theta$, being given by

$$
u_{z}=\frac{1}{2} \frac{\omega_{g}}{\omega_{p}} c .
$$

Hence one may expect a band of frequencies 0 to $1 / 2 \omega_{g}$ to be excited by the mechanism of "resonant Cerenkov radiation," this mechanism being further enhanced if the speed of the charged particles is given by the formula in (5.4).
Gallet and Helliwell [1959] point out that, if the stream intensity is sufficiently high, there may be "collective" behavior which may be regarded alternatively as a two-stream instability or as a variant of the traveling-wave amplification mechanism. The possibility that radio noise might be generated by a stream of particles entering the earth's ionosphere has also been considered by Warwick [1961].

It is also possible that a stream of high-energy particles will generate radio noise by an indirect mechanism, perhaps by the excitation of plasma oscillations in the $F 2$ region, subsequent radiation by the plasma oscillations being due to inhomogeneity or to nonlinearity.

The next energy source listed in the introduction comprises shock waves in the interplanetary medium originating on the sun. Of possible indirect mechanisms for generation of radio noise, it appears that the most important is the possibility of the excitation of whistler modes in the outer regions of the earth's magnetic field. However, there is also the possibility that there may be radiation by a more direct process, since various models for "collision-free" shock waves have been proposed [Kahn, 1955; Parker, 1959; Fishman, Kantrowitz, and Petschek, 1960] in which high-amplitude waves are generated, entropy production being accomplished by the scattering of waves rather than the scattering of particles. In this case, wave interaction processes may lead to radiation of radio noise from the shock front.

It was tempting to include in the list of energy sources rotational en ergy of the earth, since this represents such a very large store. However, it appears on closer inspection to be the least promising of the list. Within a certain neighborhood of the earth, one must expect the ambient plasma to join in of the rotational motion of the earth since the magnetic field must be "frozen" into the earth's ionosphere which rotates with the earth. Within this neighborhood, hardly any plasma process will be affected by this slow rotation. Beyond a certain region, the earth's magnetic field will have no effect so that the ambient plasma will not rotate with the earth. There is, therefore, a shear of velocity due to the earth's rotation, but this is only of the order of 1 $\mathrm{km} / \mathrm{sec}$ (the value at 10 earth's radii), so that it is completely negligible compared with the velocities ascribed to the solar wind.

Thanks are due to Dr. R. N. Bracewell, Dr. R. B. Dyce, and Dr. R. A. Helliwell for helpful conversations on topics discussed in this paper.

\section{References}

Bohm, D., and E. P. Gross, Theory of plasma oscillations. B. Excitation and damping of oscillations, Phys. Rev. 75 , 1864 (1949).

Brillouin, L., Wave propagation and group velocity (Academic Press, New York, 1960).

Fishman, F. J., A. R. Kantrowitz, and H. E. Petschek, Magnetohydrodynamic shock wave in a collision-free plasma, AVCO Research Report No. 85 (1960).

Gallet, R. M., and R. A. Helliwell, Origin of "very-low-frequency emissions," J. Research NBS 63D, 21 (1959). 
Gold, T., Plasma and magnetic fields in the solar system, J. Geophys. Research 64, 1665 (1959).

Kahn, F. D., The collision of two highly conducting ionized clouds, Gas Dynamics of Cosmic Clouds (North-Holland Publishing Company, Amsterdam, 1955), p. 115.

Lamb, H., Hydrodynamics (Cambridge University Press, London, 1930), p. 352.

Meyer, P., E. N. Parker, and J. A. Simpson, Solar cosmic rays of Feb. 1956 and their propagation through interplanetary space, Phys. Rev. 104, 768 (1958).

Parker, E. N., Dynamics of the interplanetary gas and magnetic fields, Astrophys. J. 128, 664 (1958).

Parker, E. N., Plasma dynamical determination of the shock thickness in an ionized gas, Astrophys. J. 129, 217 and 860 (1959).

Spitzer, L., Physics of fully ionized gases (Interscience, New York, N.Y., 1956), p. 53.
Storey, L. R. O., An investigation of whistling atmospherics, Phil. Trans. Roy. Soc. A246, 113 (1953).

Sturrock, P. A., In what sense do slow waves carry negative energy? J. Appl. Phys. 31, 2052 (1960).

Sturrock, P. A., Some remarks on solar radio bursts, Presented at Enrico Fermi Summer School, Varenna, Italy, May 1961(a).

Sturrock, P. A., Nonlinear effects in electron plasmas, J. Nuclear Energy-Part C: Plasma Physics 2, 158 (1961b).

Van Allen, J. A., The geomagnetically trapped corpuscular radiation, J. Geophys. Research 64, 1683 (1959).

Warwick, J. W., Observations and interpretations of atmospheric radio emission in the decametric range, Presented at URSI Conference, Washington, 1961.

(Paper 66D2-182) 